\title{
Temperature dependence of micro-deformation behavior of the porous tungsten/Zr-based metallic glass composite
}

\author{
X.Q. Zhang a , L.L. Ma ${ }^{\text {a, b }}$, Y.F. Xue ${ }^{\text {a, c, } * \text {, Q.B. Fan }}{ }^{\text {a, c }}$, Z.H. Nie ${ }^{\text {a }}$, L. Wang ${ }^{\text {a, c }}$, J.M. Yin ${ }^{\text {a }}$, H.F. \\ Zhang ${ }^{\text {d }}$, H.M. Fu ${ }^{\text {d }}$ \\ ${ }^{\text {a }}$ School of Materials Science and Engineering, Beijing Institute of Technology, Beijing 100081, \\ China. \\ ${ }^{\mathrm{b}}$ School of Chemical Engineering, Qinghai University, Xining, 810016, China. \\ ${ }^{c}$ National Key Laboratory of Science and Technology on Materials under Shock and Impact, \\ Beijing 100081, China. \\ d Shenyang National Laboratory for Materials Science, Institute of Metal Research, Chinese \\ Academy of Sciences, Shenyang 110016, China. \\ Corresponding author at: School of Materials Science and Engineering, Beijing Institute of \\ Technology, Beijing 100081, China. Tel.: +86 1068912709 ext. 107. \\ E-mail address: xueyunfei@bit.edu.cn; (Y. F. Xue)
}

\begin{abstract}
The effect of temperature on the micro-deformation behavior of the porous tungsten/Zr-based metallic glass composite was investigated during cyclic compression by synchrotron based in-situ high-energy X-ray diffraction (HEXRD) and finite element modeling (FEM). Both the metallic glass phase and the tungsten phase remained elastic in the first loading at different temperatures. The metallic glass phase also exhibits "work hardening" behavior, which is attributed to the crystallization of the metallic glass phase during deformation at high temperature. The yield strength of the tungsten phase during the second loading decreased with the
\end{abstract}


increase of temperature while remained almost equal in the third loading. Both the temperature and the pre-deformation from the cyclic loading have great influence on the work hardening behavior of the tungsten phase. The influence of the pre-deformation after the second cyclic loading is greater than the influence of the temperature during the third loading.

Key words: Metallic Glass; Composite; Temperature; high-energy X-ray diffraction.

\section{Introduction}

Bulk metallic glasses (BMGs) have many superior mechanical properties [1-3]. However, the fracture of BMGs is highly localized by shear bands during deformation, leading to nearly no macroscopic plasticity [4-6]. In order to improve the plasticity of BMGs, considerable efforts were taken to develop BMG based composites (BMGCs) [7-10]. The reinforcements of BMGCs could obstruct the rapid propagation of one major shear band and induce the formation of multiple shear bands, which are demonstrated to be responsible for enhancing the plasticity of BMGCs. The porous tungsten reinforced BMGCs exhibited work hardening behavior and excellent plasticity, which is attributed to that the porous tungsten phase could hinder the propagation of shear bands in three-dimensional (3D) directions $[11,12]$.

Temperature has great influence on the mechanical properties of materials whose structure is unstable with the change of temperature, especially for BMGs. Up to now, tremendous efforts have been devoted to investigate the deformation mechanisms of BMGs [13-20], certainly including the influence of temperature on the mechanical properties of BMGs [21-29]. However, limited literature is available on the effect of temperature on BMGCs. Qiao et al. reported that the Ti-based BMGC exhibited a decreased yield strength while an increased toughness with the increase of 
temperature [30]. Roberts et al. reported that both the Zr-based and the Ti-based BMGCs exhibited an increased yield strength but a steep decrease in toughness as the temperature decreased from the ambient temperature [31]. The present work is helpful for understanding the effect of temperature on the deformation mechanisms of the present composite, which is the key for the composite to be used in high-temperature environment. On the other hand, in order to improve the mechanical properties of BMGs, except for optimizing the composition of alloys and developing composites with different reinforced mode, deformation processing could also be available, for example, cold rolling, swaging, and hydrostatic extrusion [32-34]. Thus, the present work could also be helpful for the hot work of the present composite.

In the present study, to interpret the effect of temperature on the mechanical properties of the porous tungsten/Zr-based metallic glass composite during cyclic compression, a high energy X-ray diffraction (HEXRD) and finite element modeling (FEM) were used to investigate the micro-deformation behavior of the composite. The stress distribution and the load transferring behavior between the two phases during deformation at different temperatures were discussed in detail.

\section{Experimental}

The ingots of $\mathrm{Zr}_{41.2} \mathrm{Ti}_{13.8} \mathrm{Cu}_{12.5} \mathrm{Ni}_{10.0} \mathrm{Be}_{22.5}$ alloy were prepared by arc-melting a mixture of the five elements (the purity of the elements is above 99.5\%) in a Ti-gettered argon atmosphere. The porous tungsten with volume fraction of $80 \%$ was prepared by powder metallurgy in a hydrogen atmosphere. The details of casting could be found elsewhere [12].

An in-situ synchrotron-based HEXRD technique was employed to study the micro-deformation behavior of the composite at different temperatures. The determined temperatures are $213 \mathrm{~K}, 298 \mathrm{~K}, 423 \mathrm{~K}$, and $561 \mathrm{~K}$, respectively. The 
HEXRD experiment was performed on 11-ID-C beam line of the Advanced Photon Source, Argonne National Laboratory, USA. A monochromatic X-ray beam with energy of $115 \mathrm{keV}$ (with wavelength $0.107980 \AA$ ) was used to study the lattice strain of the tungsten phase under cyclic compression. Fig. 1 shows the experimental setup of HEXRD. The size of the rectangular specimen is $1.6 \times 1.6 \times 3.2 \mathrm{~mm}$. The ends of the specimens were polished to ensure parallelism, the lateral surfaces were also mirror polished to minimize scattering. The cyclic compression included three loading-unloading cycles. In the first loading-unloading cycle, the samples were loaded to the strain of $\sim 1 \%$. In the second and third loading-unloading cycles, the samples were loaded as far as the strain reached to $\sim 1.5$ and $\sim 3 \%$, respectively. The diffraction patterns were collected using a two-dimensional (2D) detector. The lattice strain $\varepsilon_{h k l}$ of the $\{h k l\}$ plane during loading was determined by the following equation:

$$
\varepsilon_{h k l}=\left(d_{h k l}-d_{0}\right) / d_{0}
$$

where $d$ is the interplanar spacing of the $\{h k l\}$ plane and $d_{0}$ is the interplanar spacing in the stress-free material. In the present experiment, $d_{0}$ was determined to be the initial interplanar spacing of the specimen without loading.

The interplanar spacing of the $\{h k l\}$ plane was obtained by means of Bragg's law:

$$
d_{h k l}=\lambda / 2 \sin \theta_{h k l}
$$

where $\lambda$ is the wavelength and $2 \theta_{h k l}$ is the diffraction angle from the Debye cone. $2 \theta_{h k l}$ is obtained by the individual uniaxial peak fitting.

Because the metallic glass phase is lack of the lattice strain data, FEM was employed to characterize the micro-mechanical behavior of the metallic glass phase. Details of the FEM model could be found in Ref. [35]. Table 1 shows the parameters of the constitutive model for the two phases. The yield stress of the metallic glass 
phase for the FEM model was set to be the experimental result of the Zr-based BMG at different temperatures, while the yield stress of the tungsten phase was measured by HEXRD.

The yielding criterion for the tungsten phase was analyzed by von Mises criterion [36]. The principal strains $\varepsilon_{1}, \varepsilon_{2}$, and $\varepsilon_{3}$ were measured by HEXRD, where $\varepsilon_{1}$ is the lattice strain parallel to the loading axis, $\varepsilon_{2}$ and $\varepsilon_{3}$ are the lattice strains perpendicular to the loading axis. $\varepsilon_{2}$ is equal to $\varepsilon_{3}$. The principal stresses are obtained by the constitutive equations:

$$
\begin{gathered}
\sigma_{1}=\frac{E}{1+v} \varepsilon_{1}+\frac{\vartheta E}{(1+v)(1-2 v)}\left(\varepsilon_{1}+\varepsilon_{2}+\varepsilon_{3}\right) \\
\sigma_{2}=\sigma_{3}=\frac{E}{1+v} \varepsilon_{2}+\frac{\vartheta E}{(1+v)(1-2 v)}\left(\varepsilon_{1}+\varepsilon_{2}+\varepsilon_{3}\right)
\end{gathered}
$$

where $\sigma_{1}$ is the principal stress in the longitudinal direction (LD), $\sigma_{2}$ and $\sigma_{3}$ are the principal stresses in the transverse directions (TD). The von Mises effective stress is calculated as following:

$$
\sigma=\frac{1}{\sqrt{2}}\left[\left(\sigma_{1}-\sigma_{2}\right)^{2}+\left(\sigma_{2}-\sigma_{3}\right)^{2}+\left(\sigma_{3}-\sigma_{1}\right)^{2}\right]^{1 / 2}
$$

\section{Results}

\subsection{Macro-mechanical behavior of the composite}

Fig. 2 shows the stress-strain curves of the composite at different temperatures during cyclic compression. In the first loading-unloading cycle, the composite essentially experiences only elastic deformation at all the testing temperatures. In the second cycle, the composite shows elastic-to-plastic deformation regime at all the testing temperatures, and the yield stress decreases with the increase of temperature. In the third cycle, the yield strength shows obviously lower at the low temperature, but only slight difference at the ambient and the elevated temperatures. The composite exhibits work-hardening behavior at all the testing temperatures in the third cycle. 
3.2 Micro-mechanical behavior of the tungsten phase by HEXRD

The one-dimensional (1D) HE-XRD profiles were collected using the two-dimensional (2D) detector. Considering that the 1D HEXRD profiles of the composite exhibit the same change trend at all the testing temperatures, only the $1 \mathrm{D}$ HEXRD profiles at $561 \mathrm{~K}$ were presented, as shown in Fig. 3. The 1D HEXRD profiles obviously exhibit that the diffraction peaks of the tungsten phase with a body centered cubic (bcc) structure completely cover the broad scattering hump of the metallic glass phase. To observe the tungsten phase diffraction peaks clearly, the diffraction peaks of the (222) plane were enlarged. It can be seen from Fig. 3 that the diffraction peaks of the (222) plane shift to a higher angle with increasing loading stress and shift to a lower angle with the decrease of stress.

Fig. 4 shows the lattice strain $\varepsilon_{\{211\}}$ of the tungsten phase measured by HEXRD as a function of the applied stress along the LD and the TD. As seen in Fig. 4a, the lattice strain of the tungsten phase responds linearly to the applied stress along both the LD and the TD at different testing temperatures during the first loading, suggesting that the tungsten phase experienced only elastic deformation. The discrepancy of the lattice strain in the LD and the TD attributes to the Possion's rate. The tungsten phase experienced an elastic-to-plastic regime in the second and third loading (Fig. 4b and 4c), indicating that the tungsten phase experienced a plastic deformation. It could be seen in Fig. $4 \mathrm{~b}$ that the yield strength of the tungsten phase decreased with the increase of temperature in the second loading. Compared to the obvious work hardening behavior of the tungsten phase at $213 \mathrm{~K}$ and $298 \mathrm{~K}$ during the second loading, the tungsten phase exhibits nearly perfect elastic-plastic behavior with no work hardening at $423 \mathrm{~K}$ and $561 \mathrm{~K}$. In the third loading (Fig. 4c), the yield strength of the tungsten phase at $213 \mathrm{~K}$ is still the highest, the difference from the second 
loading is that the yield stress of the tungsten phase at other temperature are nearly equal except $213 \mathrm{~K}$. It is also worth noting that the slope of the lattice strain-applied stress curves during the plastic regime in the third loading is nearly equal at different temperatures, indicating that the tungsten phase had nearly the same work hardening capacity at different temperatures in the third loading. The work hardening behavior of the tungsten phase in the third loading is more significant than that in the second loading.

\subsection{Constitutive behavior of the two phases by FEM}

Fig. 5 shows the measured and the simulated von Mises stress of the tungsten phase as a function of the applied stress at different temperatures. As seen in Fig. 5, the stress of the tungsten phase responds linearly to the applied stress during the elastic deformation at different testing temperatures. It also could be seen in Fig. 5 that the tungsten phase exhibits an increased yield stress with increasing the loading cyclic numbers at the same temperature because of work hardening. In addition, the yield strength of the tungsten phase at $213 \mathrm{~K}$ is higher than other temperatures in the second and the third cycle. The simulated von Mises stress vs. the applied stress of the tungsten phase is in accord with the measured results by HEXRD (dot mark) at different testing temperatures, indicating that the simulated result of the tungsten phase by FEM is credible.

Fig. 6 shows the simulated stress $v s$. applied stress curves of the tungsten phase and the metallic glass phase at different temperatures. In the first loading, the metallic glass phase remained elastic, and the tungsten phase sustained more applied stress than the metallic glass phase because of the elastic mismatch between the two phases. During the second and the third loading, the metallic glass phase became to be the main bearing phase after the tungsten phase yielded at all the testing temperatures. In 
the second loading, the metallic glass phase remained elastic at $213 \mathrm{~K}$ and $298 \mathrm{~K}$ while yielded at $423 \mathrm{~K}$ and $561 \mathrm{~K}$, and the yield strength of the metallic glass phase at $423 \mathrm{~K}$ is higher than that at $561 \mathrm{~K}$. The metallic glass phase yielded at all the testing temperatures in the third loading, and the yield strength of the metallic glass decreased with the increase of temperature.

\subsection{Deformation characteristic of the composite}

Fig. 7 shows the bright-field TEM images of the composite loaded to $1310 \mathrm{MPa}$ during the third loading at different temperatures. There is no crystalline phase formed in the metallic glass phase at $213 \mathrm{~K}$ and $298 \mathrm{~K}$ (Fig. 7a and 7b), while some needle-like (marked by the red arrow) and square crystalline phases (marked by the red circle) formed in the metallic glass phase near the interface at $423 \mathrm{~K}$ and $561 \mathrm{~K}$ (Fig. 7c and 7d). However, the number of the crystalline phases is so small that their diffraction peaks cannot be found in the HEXRD profiles. Dislocations (marked by the yellow arrow) generated in the tungsten phase after cyclic loading, which is in agreement with the work hardening behavior of the tungsten phase.

Fig. 8 shows the side view of the composite loaded to $1100 \mathrm{MPa}$ and $1310 \mathrm{MPa}$ during the third loading at $213 \mathrm{~K}$ and $561 \mathrm{~K}$, respectively. It could be seen in Fig. 8(a1) that the composite exhibits nearly no micro-crack or shear bands when the stress was loaded to $1100 \mathrm{MPa}$ at $213 \mathrm{~K}$. However, when the temperature was increased to $561 \mathrm{~K}$, as seen in Fig. 8(b1), a lot of micro-cracks or slip bands formed in the tungsten phase, and shear bands appeared in the metallic glass phase. Compared to the composite loaded to $1100 \mathrm{MPa}$ at $213 \mathrm{~K}$ (Fig. $8(\mathrm{a} 1)$ ), the composite loaded to $1310 \mathrm{MPa}$ at $213 \mathrm{~K}$ (Fig. 8(a2)) exhibits obvious slip bands in the tungsten phase and a few cracks in the metallic glass phase. The composite experienced obviously greater plastic deformation when loading to $1310 \mathrm{MPa}$ at $513 \mathrm{~K}$, as shown in Fig. 8(b2), the side 
surface of the composite fluctuated, remarkable cracks formed in the tips of the tungsten phase, while the interfaces between the two phases did not separate.

\section{Discussion}

\subsection{Mechanical behavior of the tungsten phase}

Fig. 9 shows the yield stress and work hardening exponent $n$ of the tungsten phase with the testing temperatures during the second and the third loading. As seen in Fig. 9, the tungsten phase exhibits a decreased yield stress with the increase of temperature in the second loading, while the tungsten phase exhibits almost equal yield stress except that at $213 \mathrm{~K}$ during the third loading. The yield strength of the metallic glass phase decreased sharply when the testing temperature near $T_{\mathrm{g}}[26]$. During the second loading at high temperatures, the metallic glass phase became soft. Thus, the metallic glass phase may have relatively weak ability to sustain the deformed tungsten phase, resulting in that reduced the yield strength of the tungsten phase decreased. However, the yield strength of the tungsten phase kept nearly constant in the third loading at temperatures above $213 \mathrm{~K}$, which is attributed to that the influence of the metallic glass phase softening on the strength of the tungsten phase is not as high as the influence of work hardening of the tungsten phase itself. Both the metallic glass phase and the tungsten phase show an increased strength when the temperature decreased to 213K. The lack of dislocation nucleation and mobility at low temperatures made the tungsten phase hard to deform [37]. The yield stress of the tungsten phase in the second loading at $213 \mathrm{~K}$ is only a little higher than that in the third loading, because several larger-scale cracks formed in the tungsten phase during the third loading, which offsets the work hardening of the tungsten phase.

The work hardening exponent, $n$, could be estimated based on the Hollomon equation [38]: 


$$
\sigma=K \varepsilon^{n}
$$

where $\sigma, K$, and $\varepsilon$ are true stress, strength factor, and true strain, respectively. As seen in Fig. 9, the work hardening exponent $n$ of the tungsten phase during the second loading decreased with the testing temperature increased from $213 \mathrm{~K}$ to $561 \mathrm{~K}$. However, the work hardening exponent $n$ of the tungsten phase during the third loading is close to a constant of 0.67 at all the testing temperatures. The different response of the work hardening behavior referred above is due to the combined action of the pre-deformation by the cyclic loading and the different deformation temperatures, the work hardening and softening competed with each other. In the second loading, the pre-deformation from the first loading was nearly completely released after the first unloading. Therefore, the work hardening exponent $n$ decreased with the increase of temperature in the second loading. During the third loading, the influence of the pre-deformation is greater than the softening at high temperatures, which caused the work hardening exponent $n$ of the tungsten phase kept nearly constant at all the testing temperatures.

\subsection{Mechanical behavior of the metallic glass phase}

The simulated stress $v s$. applied stress curves of the tungsten phase and the metallic glass phase are shown in Fig. 6. In the first loading, the tungsten phase sustained more stress than the metallic glass phase because of elastic mismatch. Fig. 10 shows the stress vs. applied stress curves of the metallic glass phase at different testing temperatures in the second and the third loading. In the second loading, the metallic glass phase remained elastic at $213 \mathrm{~K}$ and $298 \mathrm{~K}$ while yielded at $423 \mathrm{~K}$ and $561 \mathrm{~K}$, respectively. The yield strength of the metallic glass phase is still higher than that of the tungsten phase at all the testing temperatures. In contrast, the metallic glass phase yielded at all the testing temperatures and the yield strength of the metallic glass 
phase decreased with the increase of temperature during the third loading. The metallic glass phase exhibited a deformation mechanism transition from an inhomogeneous to a homogeneous mode. The flow stress could be expressed as:

$$
\sigma=\mathrm{a} E\left(\frac{\mathrm{b}-T}{T_{g}}\right)
$$

where $T$ is the testing temperature, $a$ and $b$ are constants. Thus, the metallic glass phase shows a general tendency that the flow stress decreased with the increase of temperature.

The yield strength of the metallic glass phase at $423 \mathrm{~K}$ and $561 \mathrm{~K}$ during the third loading is greater than that in the second loading, indicating that the approximate "work hardening" behavior occurred in the metallic glass phase. It is seen in Fig. 7(c-d) that crystalline phases precipitated in the metallic glass phase at $423 \mathrm{~K}$ and 561 K high temperatures. Qiao et al. [39] reported that the work hardening capacity of the $\mathrm{Zr}$-based metallic glass matrix composite is highly dependent on the plastic deformation of the crystalline phase and yield strength of the metallic glass phase. When the plastic deformation of the crystalline phase is large, as well as the yield strength of the metallic glass phase is high, the metallic glass matrix containing the crystalline phase may exhibited obvious work hardening behavior. Although the metallic glass phase started to become viscoelastic [40] at $0.7 T_{\mathrm{g}}(\sim 561 \mathrm{~K})$, the existence of the tungsten phase could still reinforce the softening metallic glass phase by means of resisting the deformation of the metallic glass phase. Thus, the flow stress of the metallic glass phase in the third loading at high temperature is greater than that in the second loading.

It should be noted that although the yield strength of the metallic glass phase decreased with the increase of temperature in the second and the third loading, the flow stress of the metallic glass phase is still higher than that of the tungsten phase. 
Thus, the metallic glass phase is still the main bearing phase after the tungsten phase yielded at all the testing temperatures.

\subsection{Interphase stress between the two phases}

Fig. 8(a1) shows that nearly no cracks formed in both the tungsten phase and the metallic glass phase when the composite was loaded to $1100 \mathrm{MPa}$ in the third loading at $213 \mathrm{~K}$. When the applied stress reached to $1310 \mathrm{MPa}$ under the same conditions, some large-scale cracks (marked by the black arrows) appeared in the tungsten phase, as shown in Fig. 8(a2). It is believed that the vibration of atoms reduced as the temperature decreased, thus both the Peierls stress and the yield strength of the tungsten phase increased as a result of the great atomic bond strength. Moreover, the screw dislocation propagation is the most important factor for the deformation of bcc metal at low temperature [41]. The driving force of screw dislocation is higher than that of edge dislocation, so the deformation of the tungsten phase is difficult to start at $213 \mathrm{~K}$, resulting in the increase of strength for the tungsten phase at low temperature. The metallic glass phase failed mainly at the tips or the interfaces where the stress concentrated at low temperature.

Compared to the composite loaded to $1100 \mathrm{MPa}$ at $213 \mathrm{~K}$ in the third loading, the composite loaded to $1100 \mathrm{MPa}$ at $561 \mathrm{~K}$ exhibits obviously greater deformation (Fig. 8(b1)). When the composite was loaded to $1310 \mathrm{MPa}$ in the third loading, both the metallic glass phase and the tungsten phase show great plasticity at $561 \mathrm{~K}$ (Fig. 8(b2)), exhibiting relatively homogeneous deformation. Numerous microcracks and slip bands formed near the interfaces in the tungsten phase, and abundant shear bands initiated in the metallic glass phase and propagated into the interfaces and tips. Because the metallic glass phase yielded relatively easy at these stress concentration regions, thus the shear bands are easy to break the resistance of the interfaces and 
propagate into the tungsten phase, which accelerated the yielding of the tungsten phase. However, the defects in the two phases intersected with each other during propagation, leading the composite to exhibit considerable plasticity at high temperature.

\section{Conclusion}

The micro-deformation behavior of the porous tungsten/Zr-based metallic glass composite was investigated under cyclic compression at different temperatures by synchrotron based in-situ high-energy X-ray diffraction (HEXRD) and finite element modeling (FEM). The main results are listed as follows:

(1) Both the tungsten phase and the metallic glass phase remained elastic during the first loading at all the testing temperatures. The tungsten phase exhibits decreased yield strength with the increase of temperature in the second loading. However, during the third loading, the tungsten phase had nearly equal yield stress from $298 \mathrm{~K}$ to $561 \mathrm{~K}$ except greater yield stress at $213 \mathrm{~K}$. Both the temperature and the cyclic loading have great influence on the work hardening behavior of the tungsten phase during the cyclic loading. By comparison, the influence of the pre-deformation from the second cyclic loading on the work hardening of the tungsten phase is greater than the influence of the temperature in the third loading.

(2) The metallic glass phase remained elastic at low temperature while yielded at high temperature during the second loading. By contrast, the metallic glass phase yielded at all the testing temperatures during the third loading, and the yield strength decreased with the increase of temperature. On the other hand, the metallic glass phase exhibits increased yield strength with the increase of the cyclic loading numbers at high temperatures, indicating that the approximate "work hardening" behavior occurred in the metallic glass phase. 
(3) At high temperature, the metallic glass phase yielded relatively easy at the interfaces between the two phases or at the tips of the metallic glass phase. Thus, the shear bands are easy to break the resistance of the interfaces and propagate into the tungsten phase, which accelerated the yielding of the tungsten phase. The two phases exhibit relatively homogeneous deformation at high temperature.

\section{Acknowledgements}

This work is supported by National Natural Science Foundation of China (Grant Nos. 51471035, 51101018, and 51271036), Hundred Talents Program of the Chinese Academy of Sciences, and Beijing Higher Education Young Elite Teacher Project. The use of the Advanced Photon Source was supported by the US Department of Energy, Office of Science Laboratory.

\section{References}

[1] W.-H. Wang, C. Dong, C. Shek, Bulk metallic glasses, Materials Science and Engineering: R: Reports, 44 (2004) 45-89.

[2] Q. Wang, Y. Yang, H. Jiang, C. T. Liu, H. H. Ruan, J. Lu, Superior tensile ductility in bulk metallic glass with gradient amorphous structure, Scientific Reports, 4 (2014) 4757.

[3] W.L. Johnson, Bulk amorphous metal—An emerging engineering material, JOM, 54 (2002) 40-43.[4] Z.F. Zhang, J. Eckert, L. Schultz, Difference in compressive and tensile fracture mechanisms of $\mathrm{Zr} 59 \mathrm{Cu} 20 \mathrm{Al10Ni8Ti3}$ bulk metallic glass, Acta Materialia, 51 (2003) 1167-1179.

[5] A. Inoue, Stabilization of metallic supercooled liquid and bulk amorphous alloys, Acta materialia, 48 (2000) 279-306.

[6] Z. Zhang, G. He, J. Eckert, L. Schultz, Fracture mechanisms in bulk metallic 
glassy materials, Physical review letters, 91 (2003) 045505.

[7] S.F. Guo, L. Liu, N. Li, Y. Li, Fe-based bulk metallic glass matrix composite with large plasticity, Scripta Materialia, 62 (2010) 329-332.

[8] D.C. Hofmann, J.-Y. Suh, A. Wiest, G. Duan, M.-L. Lind, M.D. Demetriou, W.L. Johnson, Designing metallic glass matrix composites with high toughness and tensile ductility, Nature, 451 (2008) 1085-1089.

[9] J. Mu, Z.W. Zhu, R. Su, Y.D. Wang, H.F. Zhang, Y. Ren, In situ high-energy X-ray diffraction studies of deformation-induced phase transformation in Ti-based amorphous alloy composites containing ductile dendrites, Acta Materialia, 61 (2013) 5008-5017.

[10] X. H. Sun, J. W. Qiao, Z. M. Jiao, Z. H. Wang, H. J. Yang, B. S. Xu, An improved tensile deformation model for in-situ dendrite/metallic glass matrix composites, Scientific Reports, 5 (2015) 13964.

[11] H. Zhang, A. Wang, H. Li, W. Sun, B. Ding, Z. Hu, H. Cai, L. Wang, W. Li, Quasi-static compressive property of metallic glass/porous tungsten bi-continuous phase composite, Journal of materials research, 21 (2006) 1351-1354.

[12] Y.F. Xue, H.N. Cai, L. Wang, F.C. Wang, H.F. Zhang, Dynamic compressive deformation and failure behavior of $\mathrm{Zr}$-based metallic glass reinforced porous tungsten composite, Materials Science and Engineering: A, 445-446 (2007) 275-280. [13] G. Wang, N. Mattern, J. Bednarčik, R. Li, B. Zhang, J. Eckert, Correlation between elastic structural behavior and yield strength of metallic glasses, Acta Materialia, 60 (2012) 3074-3083.

[14] G. Wang, N. Mattern, S. Pauly, J. Bednarčik, J. Eckert, Atomic structure evolution in bulk metallic glass under compressive stress, Applied physics letters, 95 (2009) 251906. 
[15] N. Mattern, J. Bednarčik, S. Pauly, G. Wang, J. Das, J. Eckert, Structural evolution of $\mathrm{Cu}-\mathrm{Zr}$ metallic glasses under tension, Acta Materialia, 57 (2009) 4133-4139.

[16] X. D. Wang, J. Bednarčik, H. Franz, H. B. Lou, Z. H. He, Q. P. Cao, J. Z. Jiang, Local strain behavior of bulk metallic glasses under tension studied by in situ x-ray diffraction, Applied physics letters, 94 (2009) 011911.

[17] M. Stoica, J. Das, J. Bednarčik, H. Franz, N. Mattern, W. H. Wang, J. Eckert Strain distribution in Zr64.13Cu15.75Ni10.12A110 bulk metallic glass investigated by in situ tensile tests under synchrotron radiation, Journal of applied physics, 104 (2008) 013522.

[18] J. Das, M. Boström, N.Mattern, Å. Kvick, A.R. Yavari, A.L. Greer, J. Eckert. Plasticity in bulk etallic glasses investigated via the strain distribution, Phys. Rev. B, 76 (2007) 092203.

[19] T. C. Hufnagel, R. T. Ott, J. Almer, Structural aspects of elastic deformation of a metallic glass, Phys. Rev. B 73 (2006) 064204.

[20] H.F. Poulsen, J.A. Wert1, J.Neuefeind, V. Honkimäki, M. Daymond, Measuring strain distributions in amorphous materials, Nature Materials, 4 (2005) 33-36.

[21] W.D. Liu, K.X. Liu, Mechanical behavior of a Zr-based metallic glass at elevated temperature under high strain rate, Journal of Applied Physics, 108 (2010) 033511.

[22] G. Wang, J. Shen, J.F. Sun, Z.P. Lu, Z.H. Stachurski, B.D. Zhou, Tensile fracture characteristics and deformation behavior of a Zr-based bulk metallic glass at high temperatures, Intermetallics, 13 (2005) 642-648.

[23] Y. Huang, J. Shen, J. Sun, Z. Zhang, Enhanced strength and plasticity of a Ti-based metallic glass at cryogenic temperatures, Materials Science and Engineering: A, 498 (2008) 203-207. 
[24] Y. Kawamura, K.B. Kim, Superplastic forming of zr65A110Ni10Cu15 metallic glass, in: Materials science forum, Materials science forum, 1999, pp. 373-378.

[25] W.J. Kim, D.S. Ma, H.G. Jeong, Superplastic flow in a Zr65Al10Ni10Cu15 metallic glass crystallized during deformation in a supercooled liquid region, Scripta Materialia, 49 (2003) 1067-1073.

[26] K.S. Lee, T.K. Ha, S. Ahn, Y.W. Chang, High temperature deformation behavior

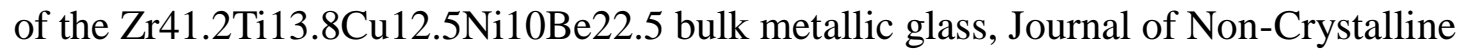
Solids, 317 (2003) 193-199.

[27] H. Li, C. Fan, K. Tao, H. Choo, P.K. Liaw, Compressive Behavior of a Zr-Based Metallic Glass at Cryogenic Temperatures, Advanced Materials, 18 (2006) 752-754.

[28] A. Kawashima, Y. Zeng, M. Fukuhara, H. Kurishita, N. Nishiyama, H. Miki, A. Inoue, Mechanical properties of a Ni60Pd20P17B3 bulk glassy alloy at cryogenic temperatures, Materials Science and Engineering: A, 498 (2008) 475-481.

[29] J. Xu, U. Ramamurty, E. Ma, The fracture toughness of bulk metallic glasses, JOM, 62 (2010) 10-18.

[30] J.W. Qiao, Y. Zhang, H.L. Jia, H.J. Yang, P.K. Liaw, B.S. Xu, Tensile softening of metallic-glass-matrix composites in the supercooled liquid region, Applied Physics Letters, 100 (2012) 121902.

[31] S. Roberts, C. Zachrisson, H. Kozachkov, A. Ullah, A.A. Shapiro, W.L. Johnson, D.C. Hofmann, Cryogenic Charpy impact testing of metallic glass matrix composites, Scripta Materialia, 66 (2012) 284-287.

[32] Y. F. Xue, H. N. Cai, L. Wang, F. C. Wang H. F. Zhang, Strength-improved Zr-based metallic glass/porous tungsten phase composite by hydrostatic extrusion [J]. Applied Physics Letter, 2007, 90: 8.

[33] Y.F. Xue, H.N. Cai, L. Wang, F.C. Wang, H.F. Zhang, Z.Q. Hu, Deformation and 
failure behavior of a hydrostatically extruded Zr38Ti17Cu10.5Co12Be22.5 bulk metallic glass/porous tungsten phase composite under dynamic compression $[\mathrm{J}]$. Composites science and technology, 2008, 68: 3392-3400.

[34] Y.F. Xue, L. Wang, H.W. Cheng, F.C. Wang, H.F. Zhang, Shear band formation and mechanical properties of $\mathrm{Zr} 38 \mathrm{Ti} 17 \mathrm{Cu} 10.5 \mathrm{Co} 12 \mathrm{Be} 22.5$ bulk metallic glass/porous tungsten phase composite by hydrostatic extrusion [J]. Material Science and Engineering: A, 2010, 527: 21-22.

[35] X.Q. Zhang, L. Wang, Q.B. Fan, Y.F. Xue, Y.D. Wang, Z.H. Nie, H.F. Zhang, H.M. Fu, Micro-deformation mechanism of Zr-based metallic glass/porous tungsten composite by in-situ high-energy X-ray diffraction and finite element modeling, Materials Science and Engineering: A, 598 (2014) 407-412.

[36] G.E. Dieter, D. Bacon, Mechanical metallurgy, McGraw-Hill New York, 1986.

[37] P. Gumbsch, J. Riedle, A. Hartmaier, H.F. Fischmeister, Controlling Factors for the Brittle-to-Ductile Transition in Tungsten Single Crystals, Science, 282 (1998) 1293-1295.

[38] J.H. Hollomon, Tensile deformation, AIME TRANS, 12 (1945) 1-22.

[39] J.W. Qiao, A.C. Sun, E.W. Huang, Y. Zhang, P.K. Liaw, C.P. Chuang, Tensile deformation micromechanisms for bulk metallic glass matrix composites: From work-hardening to softening, Acta Materialia, 59 (2011) 4126-4137.

[40] Q. Wang, D.K. Wang, T. Fu, J.J. Blandin, J.M. Pelletier, Y.D. Dong, High temperature homogeneous plastic flow behavior of a $\mathrm{Zr}$ based bulk metallic glass matrix composite, Journal of Alloys and Compounds, 495 (2010) 50-54.

[41] P. Ratchev, B. Vandevelde, B. Verlinden, B. Allaert, D. Werkhoven, Brittle to Ductile Fracture Transition in Bulk Pb-Free Solders, Components and Packaging Technologies, IEEE Transactions on, 30 (2007) 416-423. 
Table 1

Table 1 Material parameters

\begin{tabular}{|c|c|c|c|c|}
\hline Material & \multicolumn{2}{|c|}{$\sigma_{\mathrm{y}}(\mathrm{MPa})$} & $E(\mathrm{GPa})$ & $v$ \\
\hline Zr-based metallic & 2000 (213K) & $1900(298 \mathrm{~K})$ & \multirow{2}{*}{96} & \multirow{2}{*}{0.36} \\
\hline glass & $1600(423 \mathrm{~K})$ & $1380(561 \mathrm{~K})$ & & \\
\hline Tungsten & \multicolumn{2}{|c|}{ measured by HEXRD } & 410 & 0.28 \\
\hline
\end{tabular}

